Research Paper

\title{
MiR-34a in Age and Tissue Related Radio-Sensitivity and Serum miR-34a as a Novel Indicator of Radiation Injury
}

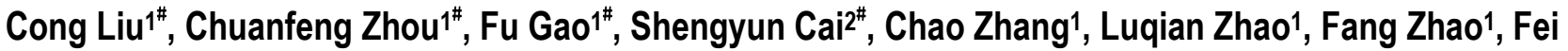

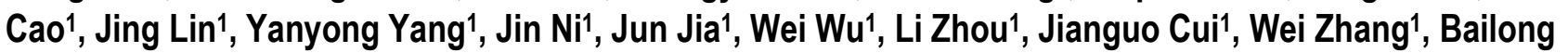 \\ $\mathrm{Li}^{1 凶}$, Jianming Cai ${ }^{1 凶}$
}

1. Department of Radiation Medicine, Faculty of Naval Medicine, Second Military Medical University, Xiangyin Road, 200433, Shanghai, PR China;

2. Department of Obstetrics and Gynecology, Changhai Hospital, Second Military Medical University, Shanghai 200433, China.

\# These authors contributed equally to this work.

Corresponding author: Jianming Cai \& Bailong Li, Department of Radiation Medicine, Second Military Medical University; 800, Xiangyin Road 200433, Shanghai, P.R. China. Fax: +86-21-81871150. E-mail: caijianming882003@163.com \& bailongli2010@163.com.

(C) Ivyspring International Publisher. This is an open-access article distributed under the terms of the Creative Commons License (http://creativecommons.org/ licenses/by-nc-nd/3.0/). Reproduction is permitted for personal, noncommercial use, provided that the article is in whole, unmodified, and properly cited.

Received: 2010.09.30; Accepted: 2011.03.01; Published: 2011.03.02

\begin{abstract}
MiR-34a, a direct target of $\mathrm{p} 53$, has shown to exert potent anti-proliferative effects. It has also been found that miR-34a can be induced by irradiation in vitro and in vivo. However, the relationship between miR-34a and radio-sensitivity, and its potential diagnostic significance in radiation biology, remain unclear. This study found that differing responses to ionizing radiation (IR) of young and adult mice were related to miR-34a. First, we found that miR-34a could be induced in many organs by radiation of both young and adult mice. However, the level of miR-34a induced by young mice was much higher when compared to adult mice. Next, we found that miR-34a played a critical role in radio-sensitivity variations of different tissues by enhancing cell apoptosis and decreasing cell viability. We also found that the induction of miR-34a by radiation was in a $\mathrm{p} 53$ dependent manner and that one possible downstream target of miR-34a that lead to different radio-sensitivity was the anti-apoptosis molecular $\mathrm{Bcl}-2$. However, over-expression of miR-34a and knockdown of $\mathrm{Bcl}-2$ could significantly enhance the radio-sensitivity of different cells while inhibition of miR-34a could protect cells from radiation injury. Finally, we concluded that miR-34a could be stable in serum after IR and serve as a novel indicator of radiation injury. Taken together, this data strongly suggests that miR-34a may be a novel indicator, mediator and target of radiation injury, radio-sensitivity and radioprotection.
\end{abstract}

Key words: Radio-sensitivity, MicroRNA,MiR-34a, P53, Bcl-2, Apoptosis.

\section{Introduction}

MicroRNAs (miRNAs,miRs) are a novel class of highly conserved, small, non-coding RNAs, about 22 to 25 nucleotides in length, that have been shown to regulate gene expression in many important pathways in many organisms $[1,2]$. These RNA molecules are produced from larger transcripts that are processed by RNase III enzyme Drosha/Pasha and Dicer [3]. Studies showed that Drosha/Pasha and Dicer work se- 
quentially to generate mature 20-25 nucleotide miRNAs from the hairpin precursors [1]. They function by binding to the 3'UTR of multiple target mRNAs [4-6]. The bound mRNAs resulted in a decreased number of transcripts [7]. In animal cells, miRNAs play a vital role in development, cell proliferation, differentiation, apoptosis, and metabolism [8-10]. However, the role of miRNAs in radiation biology is unclear and only about 10 papers have been published on ionizing radiation (IR) induced miRNA [11]. Two papers attracted our attention were the change of miR-34a after IR $[11,12]$, and its potent anti-proliferative effects and ability to induce cell apoptosis [12-14]. It is well established that miR-34a is a direct target of p53, whose induction by DNA damage depends on p53 [11]. Besides, the role of p53 in the radio-sensitivity of different cells and tissues has been extensively studied [15-17]. It is well accepted that wild-type p53 is one of the key factors that enhanced the radio-sensitivity of many cells $[16,18]$ while the p53-mutated or deficient cells, especially some p53 deficient cancer cells, respond very poorly to radiation $[19,20]$. However, the role of miR-34a in age and tissue related radio-sensitivity and its' potential diagnostic significance in radiation biology, remain unclear.

The relationship between radio-sensitivity and age has been recognized for many years [21-23]. Using a single dose, Abrams found that age, body weight, and sex could influence radiation susceptibility[21]. The Sacher group and Crosfill group studied the relationship between radio-sensitivity and age systematically and found that young mice have a higher radio-sensitivity than adult mice $[22,23]$. Similar results were obtained by the Rotblat group [24, 25]. In 1971, Chaffey and Hellman found that differing responses to IR of murine bone marrow stem cells were related to the cell cycle[26]. However, in all of these studies the mechanism of age dependent radio-sensitivity was largely ignored and the role of miRNA in radio-sensitivity was given even less study.

In this study, we first found that miR-34a could be induced in many organs by the radiation of both young and adult mice. However, the level of miR-34a induced by young mice was much higher when compared to adult mice, which was closely correlated with the fact that young mice had a higher radio-sensitivity when compared to adult mice. Next, we found that miR-34a correlated closely with radio-sensitivity variations of different tissues in genetic normal mice. However, the level of miR-34a induced by IR seems to be in a p53 dependent manner since the level of miR-34a induced by IR in HCT116 p53\%- cells was decreased significantly when compared to HCT116 p53 $3^{+/+}$cells. Then, we used transfection assay to find that over-expression of miR-34a markedly suppressed cell proliferation and enhanced apoptosis both before and after radiation. Furthermore, we also found that the possible downstream target of miR-34a that led to different radio-sensitivity was $\mathrm{Bcl}-2$ since the over-expression of miR-34a and the knockdown of Bcl-2 could significantly enhance the radio-sensitivity of different cells. Finally, we found that miR-34a could be detected not only in tissues, but also in mice serum, while the p53 mRNA could not be detected in mice serum after IR, which indicated miR-34a is a novel indicator of radiation injury. Taken together, our data demonstrated that miR-34a may be a novel indicator, mediator and target of radiation injury, radio-sensitivity and radioprotection.

\section{Materials and Methods}

\section{Experimental animals and grouping}

Thirty young (3-4 weeks adult) and thirty adult (6-8 weeks adult) male BALB/c mice or C57BL/6 mice were purchased from Chinese academy of science (Shanghai, China). All mice were housed in a pathogen-free facility for all experiments. All animal experiments were undertaken in accordance with the National Institute of Health Guide for the Care and Use of Laboratory Animals, with the approval of the Laboratory Animal Center of Second Military Medical University, Shanghai. The approval ID for this study was 20081011. All efforts were made to minimize the number of animals used as well as their suffering.

\section{Cell culture}

Human glioblastoma-astrocytoma, epithelial-like cell line U87MG cells or Human Embryonic Kidney 293 cells HEK293 cells were obtained from the Shanghai Institute of Biochemistry and Cell Biology, Chinese Academy of Sciences (Shanghai, China). HCT116 p53-/- cells, HCT116 p53+/+ cells were cultured in 24 -well plates at $37^{\circ} \mathrm{C}$ in a humidified atmosphere of $5 \% \mathrm{CO} 2$ with DMEM Medium (PAA, Austria) containing $10 \%$ fetal calf serum (PAA, Austria). Exponentially growing cells were used for experiments.

\section{Total-body irradiation}

A ${ }^{60} \mathrm{Co}$ irradiator was introduced for total-body ionizing irradiation as described in our previously studies [27]. In detail: un-anaesthetized mice were placed in well-ventilated plastic boxes and exposed to the ${ }^{60} \mathrm{Co}$ irradiator at a distance of $3 \mathrm{~m}$ from the source. A single dose of 7 Gy (or 2Gy and12 Gy) $\gamma$-radiation was delivered depending upon the requirement of the study. The dose rate was $1 \mathrm{~Gy} / \mathrm{min}$ in this study. 


\section{Survival Experiments}

Mice were irradiated with the lethal doses described above, and the survival was recorded daily up to day 30 after irradiation. Differences in survival of the various groups of mice were assessed using Kaplan-Meier analyses and tested for significant differences using the Log Rank test. Changes in median survival (days at risk after exposure or sham exposure, +/- S. E.) with increasing dose were derived from the Kaplan-Meier analyses and tested by ANOVA for consistency with linear regression analyses.

\section{RNA extraction}

RNA from different spleens, livers, thymus and cell lines was homogenized in Trizol (Invitrogen) and isolated according to the manufacturer's instructions as described previously [11]. RNA from serums was homogenized in mirVana ${ }^{\mathrm{TM}}$ PARISTM Kit (Part Number AM1556, Applied Biosystems, USA) according to the manufacturer's instructions. Concentration and purity of total RNA samples were measured using the SmartSpec Plus spectrophotometer (Bio-Rad, Hercules, CA). The ratio of A260:A280 was used to indicate the purity of total RNA.

\section{Reverse Transcription}

cDNA was generated using the miScript Reverse Transcription (RT) Kit (Qiagen GmbH, Hilden, Germany). According to the manufacturer's instructions, 1ug total RNA, 1ul miScript Reverse Transcriptase Mix, and 4ul miScript RT buffer were mixed well and incubated for $60 \mathrm{~min}$ at $37 \mathrm{C}$. All reverse transcriptions and no-template controls were run at the same time.

\section{Real-Time PCR}

Real-time polymerase chain reaction (PCR) was performed using miScript SYBR Green PCR Kit (Qiagen) on a Rotorgene 3000 series PCR machine (Corbett Research) and analyzed with software of Rotorgene System. The 20ul PCR mixture included 2ul reverse transcription product, 10ul 2× QuantiTect SYBR Green PCR Master Mix, 2ul 10× miScript Universal Primer, 2ul 10× miScript Primer Assay (for miR-34a; Qiagen), and 4ul autoclaved distilled water. The reaction mixtures were incubated at $95^{\circ} \mathrm{C}$ for $10 \mathrm{~min}$, followed by 40 amplification cycles of $94 \mathrm{C}$ for $15 \mathrm{~s}, 55 \mathrm{C}$ for $30 \mathrm{~s}$, and $70 \mathrm{C}$ for $30 \mathrm{~s}$. The threshold cycle $(\mathrm{Ct})$ was defined as the fractional cycle number at which the fluorescence passed the fixed threshold. We also quantified transcripts of U6 small RNA using Hs_RNU6B_2 miScript Primer Assay (Qiagen) for normalizing the levels of miR-34a. Small RNAs are preferred because they have similar purification and amplification characteristics to miRNAs. Choosing an appropriate control for a miRNA quantification experiment is similar to choosing an appropriate housekeeping gene for normalization when quantifying mRNA. For each sample type or treatment under study, it is necessary to verify that the control RNA is not regulated under the experimental conditions. In addition, a control for a non-coding RNA that shows a constant level of expression and similar abundance to the target miRNA should be chosen.U6 small RNA is a widely used endogenous reference RNA in miRNA quantification studies. Serum miR-34a was normalized to miR-16 or Exogenous U6 RNA as described previously [28-30] [31].The ${ }_{\Delta} \mathrm{Ct}$ method was used for analysis. The experiment was repeated 3 times. The investigators were blinded to the results of clinical and pathological diagnosis.

\section{Transfection}

Cells were cultured and transfected as described previously [32]. Human glioblastoma-astrocytoma cell line U87MG cells; Human Embryonic Kidney 293 cells HEK293 cells, HCT116 p53/- cells and HCT116 p53+/+ cells were seeded at $6 \times 10^{4}$ cells per well in 24-well plates and transfected with synthetic miRNAs (mimics/inhibitors, genepharm, shanghai, china) at a final concentration of $50 \mathrm{nM}$ or $100 \mathrm{nM}$ using Lipofectamine 2000 (Invitrogen, ON, Canada) transfection reagent according to the manufacturer's instructions as described previously [32]. Three anti-Bcl-2 siRNA (siRNA 1, siRNA 2 and siRNA 3) and a control non-targeting siRNA (siRNA NC) were purchased from genepharm company (genepharm, shanghai, china) and they were transfected into cells similar to miRNA. Cells were infected $48 \mathrm{~h}$ later and subjected to further experiments.

\section{Apoptosis assay}

After different treatments, single cell suspension of Spleen, liver and thymus tissues were made and labeled with annexin V-FITC and propidium iodide (PI) provided by BIPEC, following manufacturer's instructions as described by our previously studies[27]. Samples were examined by fluorescence-activated cell sorter (FACS) analysis, and the results were analyzed using Cell-Quest software (Becton Dickinson, San Jose, CA).

\section{Cell Viability Analyses}

Trypan blue exclusion test were used for detecting cell viability as previously described in our previously studies[26]. Briefly, after different treatments, single cell suspension of spleen, liver and thymus tissues were made and labeled with Trypan Blue. The cellular morphology was observed using Olympus 
BX60 fluorescent microscope equipped with Retiga 2000R digital camera. Average percentage of Trypan Blue positives and negative cells were calculated in 5-7 randomly selected high power field (HPF). Trypan Blue negative cells were regarded as living cells.

\section{Statistical analysis}

Comparisons between experimental groups and relevant controls were performed by Student's t-test. $\mathrm{P}<0.05$ was considered a statistically significant difference.

\section{Results}

\section{MiR-34a is closely related to age related ra- dio-sensitivity}

To study the role of miR-34a in age related radio-sensitivity, we first recorded the survival ability of different aged mice after receiving a total-body irradiation (TBI) at a single dose of $7 \mathrm{~Gy}$. We found that the survival capacity of young (3-4 weeks old) mice was much lower than adult (6-8 weeks old) BALB/c mice after TBI (Figure 1A). This data indicated that young mice have a higher radio-sensitivity than adult mice. To further prove our finding, we also recorded the survival ability of different aged mice after receiving a single dose of $12 \mathrm{~Gy}$ TBI. The mean survival time of adult mice was $120 \pm 24.0 \mathrm{~h}$; while, the mean survival time was significantly decreased in young mice, which had a mean survival time of $72 \pm 36 \mathrm{~h}$ (Figure 1A). We also found that Total-body irradiation suppressed the water consumption and food intake, and resulted in weight loss in both young as well as adult mice. However, TBI suppressed young mice were much more effective than adult mice (data not shown). Taken together, our data suggests that young mice have a higher radio-sensitivity when compared to adult mice. Next, we studied the possibility of miR-34a in age related radio-sensitivity. We first used q-RT-PCR analysis to detect the relative expression of miR-34a in different tissues from different aged mice. We found that miR-34a could be induced in the spleen by radiation of both young and adult mice 8 hours after irradiation. However, the level of miR-34a induced by young mice was much higher compared to adult mice (Figure 1B). Similar data was reported in thymus tissue after 7Gy total-body irradiation (Figure 1B). The different ability to induce miR-34a by young and adult mice was closely related to the differences in their radio-sensitivity.

To further study the time and dose response of miR-34a induced by different aged mice, we then detected miR-34a level in spleen after 7Gy IR for various time periods and found that miR-34a can be induced by IR in a time-dependent manner (Figure 1C). We also detected the miR-34a level in spleen after $2 \mathrm{~Gy}$ and $12 \mathrm{~Gy}$ IR and found that 2Gy IR, although a little weaker than 7 Gy IR and 12Gy IR, was enough to induce miR-34a in spleen (Figure 1C). The time and dose response also showed that young mice have higher ability to induce miR-34a than adult mice and these data indicate that miR-34a is closely related to age related radio-sensitivity.

\section{MiR-34a was correlated to radio-sensitivity vari- ations of different tissues and mice strains.}

During our study of miR-34a in age related radio-sensitivity we found an interesting phenomenon. The relative level of miR-34a induced by IR in thymus tissues was much higher when compared to spleen tissues after TBI (Figure 1B and 2C). Indeed, radio-sensitivity variations among different tissues were also an interesting issue and the role of miRNA in this area is still not clear. Then we hypothesized that miR-34a may not only play a role in age related radio-sensitivity, but also a novel mediator of tissue related radio-sensitivity and radiation injury. To prove this theory, we first analyzed the radio-sensitivity variations among different spleen, thymus and liver tissues. We found that the spleen, thymus and liver have a similar apoptosis rate before radiation. However, the apoptosis rate of the liver is much lower than thymus after TBI, while the spleen has a middle apoptosis rate between the liver and thymus after TBI (Figure 2A and 2B); Consistent with the apoptosis data, the spleen, thymus and liver also have similar cell viability before radiation. However, the liver tissue has a much higher proliferate capacity than the thymus tissues, while the spleen has higher cell viability than the thymus but lower than the liver after TBI (Figure 2B). This data indicates that, consistent with previous studies, the thymus has a relatively high radio-sensitivity while the liver has a fairly low radio-sensitivity and the radio-sensitivity of the spleen is between the two. Next we studied the possibility of miR-34a in tissue related radio-sensitivity. Interestingly, we found that miR-34a could be induced in the thymus, liver and spleen by radiation in nearly every mouse. However, the relative level of miR-34a induced in the thymus tissue is much higher when compared to the liver after TBI (Figure 2C). Spleen tissues also have a higher capacity to induce miR-34a compared to the liver and slightly higher than the thymus after TBI. The ability to induce miR-34a by different tissues was closely related to their radio-sensitivity. These data also indicate that miR-34a is correlated to tissue related radio-sensitivity. 
A

7 Gy 12 Gy

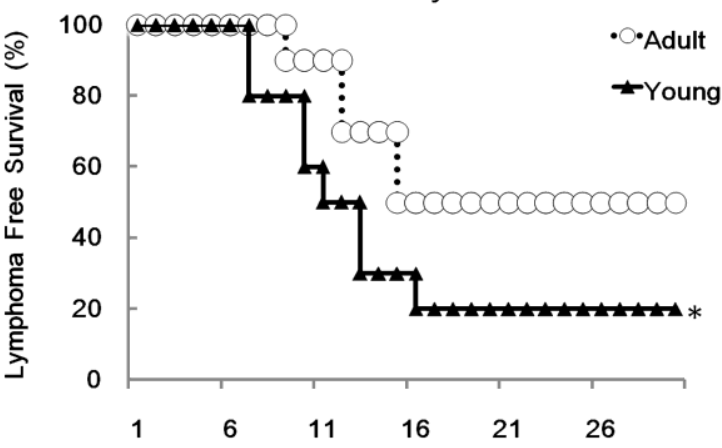

Time after Last Irradiation (Days)

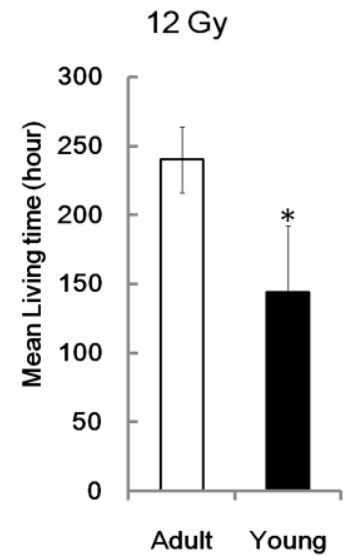

B

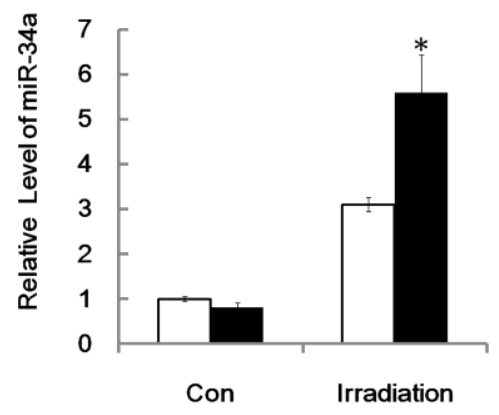

C

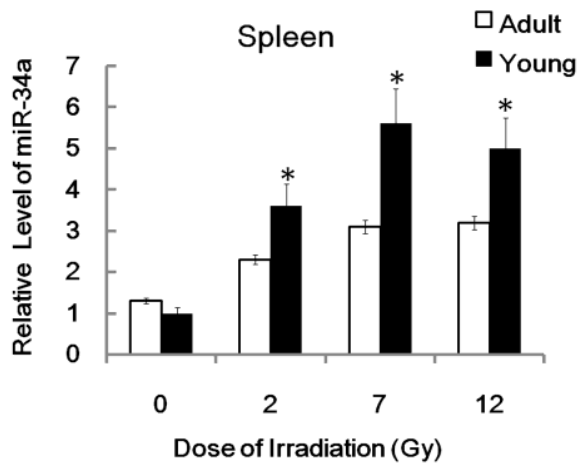

Thymus
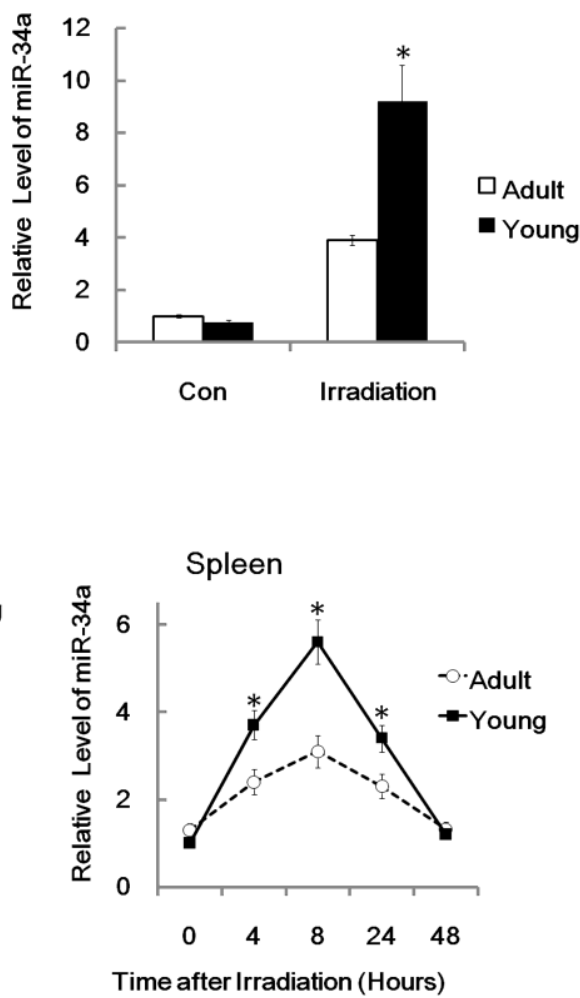

Figure I. MiR-34a in age related radio-sensitivity. (A).Left: Yong or adult BALB/c mice were irradiated (+IR; 7Gy, dose rate I Gy/min.) and survival time were recorded. Right: Survival time of mice in different groups after total-body irradiation (+IR; I2Gy, dose rate I Gy/min.). N=20. (B) Yong or adult BALB/c mice were either irradiated (+IR; 7Gy, dose rate I Gy/min) or left untreated (con) and sacrificed $8 \mathrm{hr}$ later, Spleen(left) or thymus (right) RNA was subjected to qRT-PCR analysis. N=6. (C) miR-34a can be induced by IR in a dose and time-dependent manner. Right: Yong or adult BALB/c mice were either irradiated (+IR; 7Gy, dose rate I Gy/min) or left untreated (con) and sacrificed 4,8,24 and $48 \mathrm{hr}$ later, Spleen RNA was subjected to qRT-PCR analysis. $N=6$. Left: Yong or adult BALB/c mice were either irradiated with different doses (+IR; $2 \mathrm{~Gy}$, $7 \mathrm{~Gy}$ or $12 \mathrm{~Gy}$, dose rate I Gy/min) or left untreated (con) and sacrificed $8 \mathrm{hr}$ later, Spleen RNA was subjected to qRT-PCR analysis. Levels of miR-34a were normalized in the same sample and the level of miR-34a in adult un-irradiated mice was regarded as 100\%. Data show the relative miR-34a expression level. $N=6 ; *$ : $P<0.05$. 

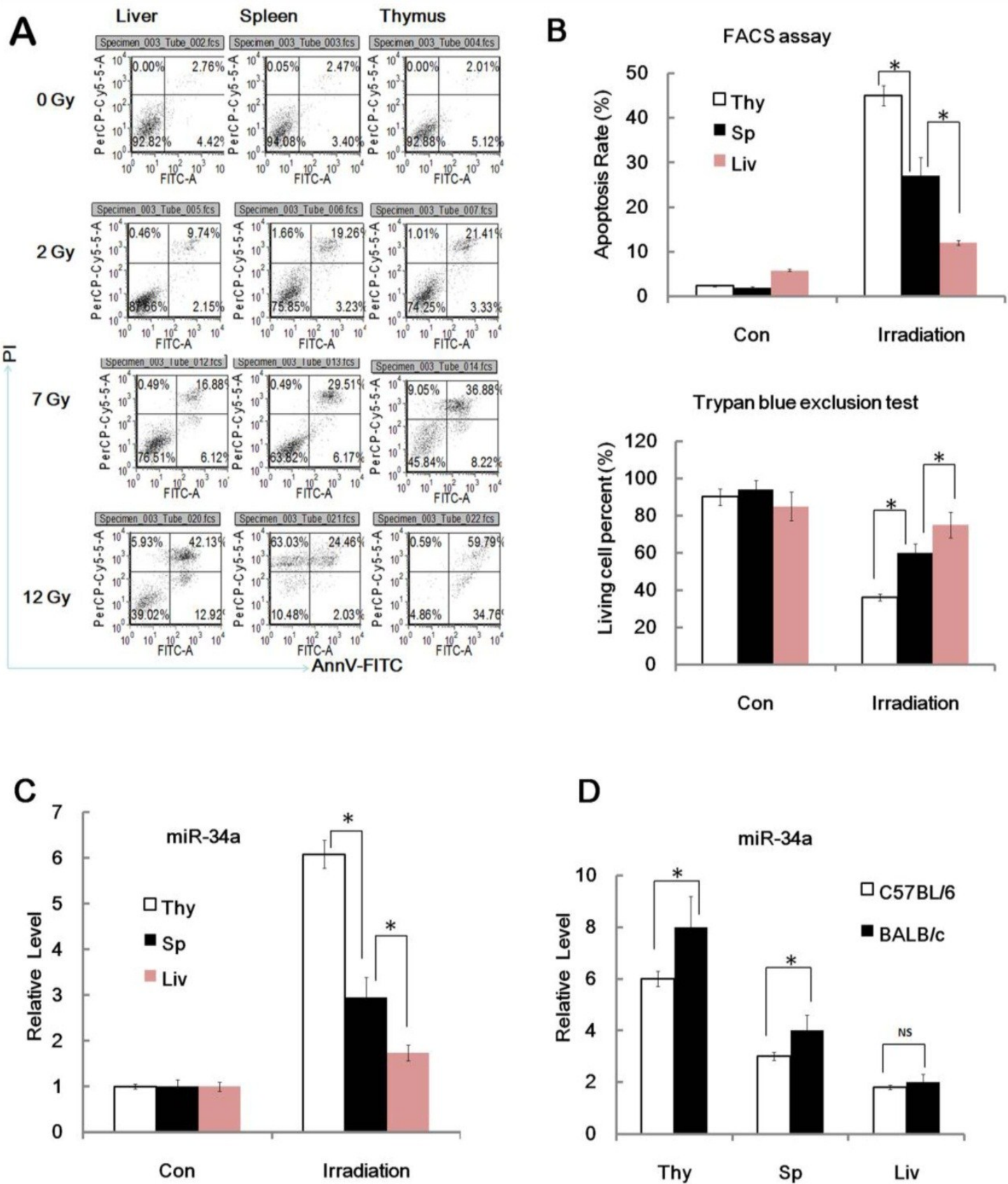

Figure 2. MiR-34a in radio-sensitivity variations of different tissues and mice strains. (A). BALB/c mice were irradiated with different doses (+IR; 2 Gy, 7 Gy or 12 Gy, dose rate I Gy/min) or left untreated (con) and sacrificed $24 \mathrm{hr}$ later. Spleen, thymus and liver cells were stained with annexin $\mathrm{V}-\mathrm{FITC}$ and $\mathrm{Pl}$ and subjected to FACS assay of cellular apoptosis. N=6. Data showed a typical data of 3 repeats. (B). upper: BALB/c mice were irradiated (+IR; 7Gy, dose rate $0.8 \mathrm{~Gy} / \mathrm{min}$.) or left untreated then sacrificed $24 \mathrm{hr}$ later for apoptosis assay. Figure showed statistical apoptosis rate of Spleen (Sp), thymus (Thy) and liver (Liv). Lower: BALB/c mice were irradiated (+IR; 7Gy, dose rate I Gy/min.) or left untreated then sacrificed $24 \mathrm{hr}$ later. Spleen, thymus and liver cells were subjected to Trypan blue exclusion test for detection of cell viability. $\mathrm{N}=6$. (C).BALB/c mice were irradiated (+IR; 7Gy, dose rate I Gy/min.) or left untreated then sacrificed $8 \mathrm{hr}$ later. Spleen, thymus and liver cells RNA was subjected to qRT-PCR analysis. Levels of miR-34a were normalized in the same sample and the level of miR-34a in adult un-irradiated mice was regarded as $100 \%$. Data show the relative miR-34a expression level. $N=6$. (D).BALB/c mice and C57BL/6 mice were irradiated (+IR; 7Gy, dose rate I Gy/min.) or left untreated then sacrificed $8 \mathrm{hr}$ later. Spleen, thymus and liver cells RNA was subjected to QRT-PCR analysis. Levels of miR-34a were normalized in the same sample and the level of miR-34a in adult un-irradiated mice was regarded as $100 \%$. Data show the relative miR-34a expression level of 7Gy compare to 0Gy in the same strain. $\mathrm{N}=6 . *: \mathrm{P}<0.05$; NS: No Significant difference detected. 
Since C57BL/ 6 mice are less sensitive to ionizing radiation than radiation sensitive strain $\mathrm{BALB} / \mathrm{c}$ mice, we then detected the miR-34a level in the thymus, liver and spleen from C57BL/6 mice and BALB/C mice before and after 7 Gy IR and found that IR can induce miR-34a expression in thymus, liver and spleen tissues from both C57BL/6 mice and BALB/c mice (Figure 2D), however, the thymus and spleen from BALB/c mice showed higher miR-34a induction, while the livers from C57BL/ 6 mice and BALB/c mice showed similar miR-34a enhancement. These data also indicate that miR-34a was correlated to radio-sensitivity variations of different mice strains.

\section{The induction of MiR-34a by IR is in a p53 de- pendent manner}

Since miR-34a is a direct target of p53 in many pathways [11], we next used p53 sufficient and deficient cell line HCT116 p53+/+ cells and HCT116 p53-/cells to study the possible relationship of miR-34a and p53 in radio-sensitivity. We first found that the radio-sensitivity of HCT116 $\mathrm{p53}^{+/+}$cells was significantly higher than HCT116 p53\%- cells since HCT116 p53 ${ }^{+/+}$cells displayed higher apoptosis cell rate and reduced cell viability than HCT116 p53\%- cells after the same dose of IR (Figure 3A and 3B).

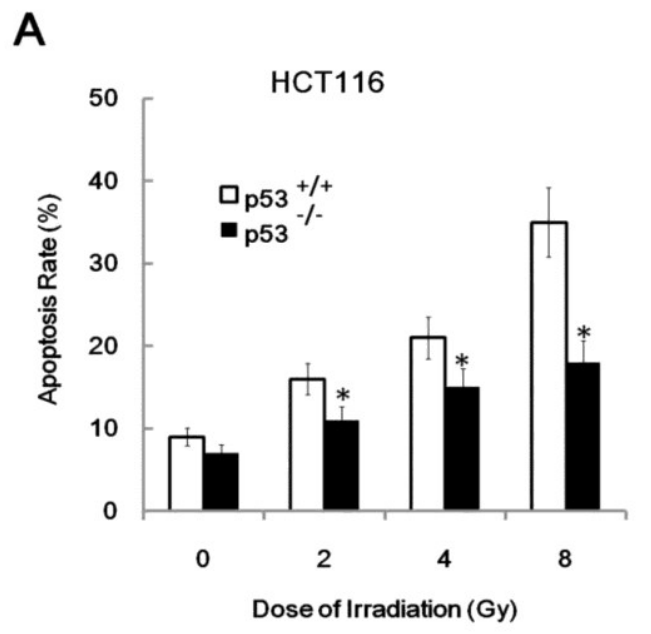

B
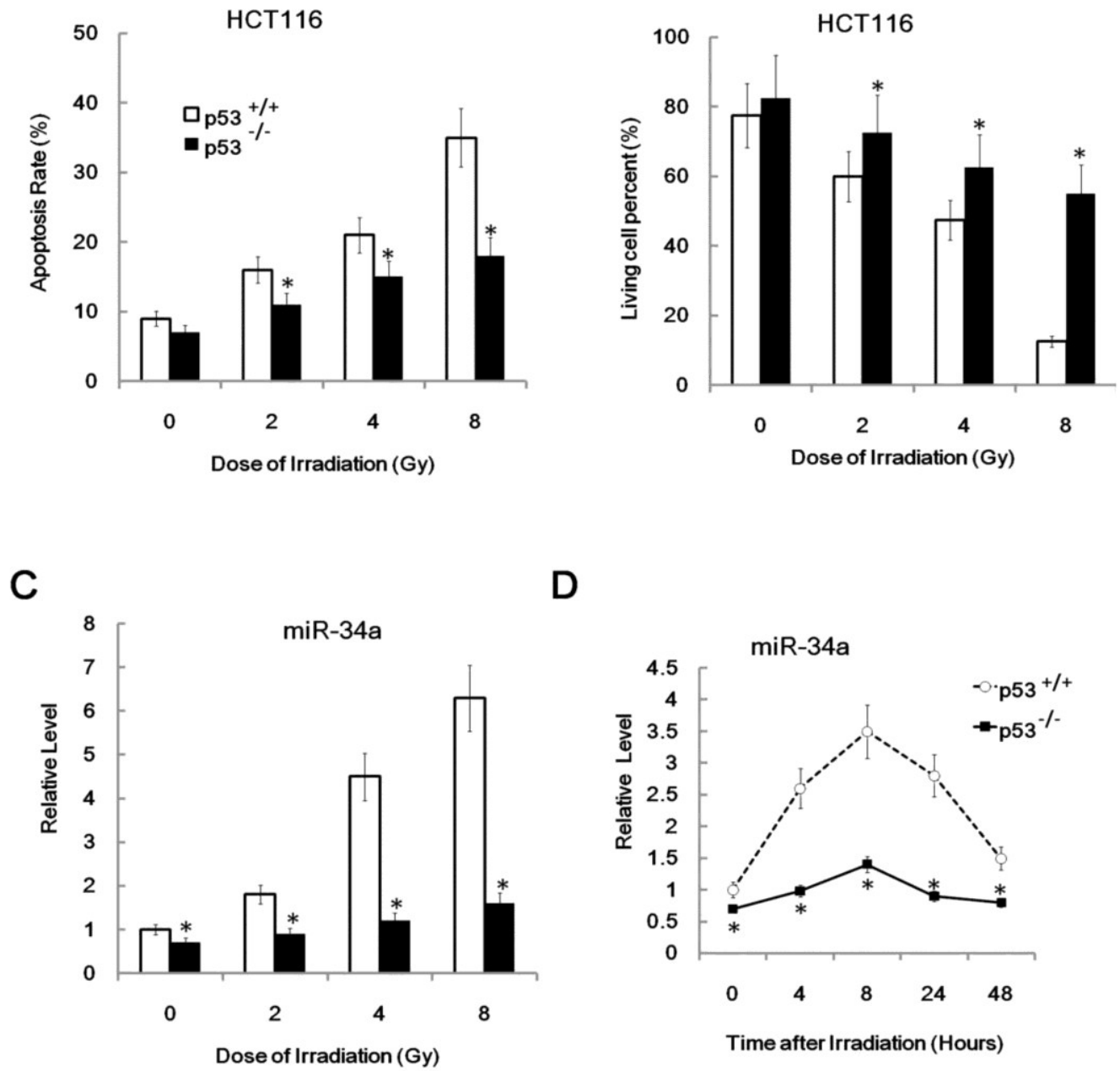

Figure 3. The induction of MiR-34a by IR is in a p53 dependent manner. (A/B). HCTII 6 p53 $3^{+/+}$cells and HCTII6 p53 $3^{-/-}$cells mice were irradiated with different doses (+IR; 2 Gy, 4 Gy or 8 Gy, dose rate I Gy/min) or left untreated (0 Gy) and 24 hr later cells were subjected to FACS assay $(A)$ of cellular apoptosis and Trypan blue exclusion test for detection of cell

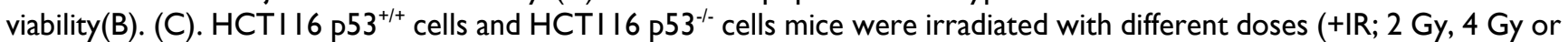
$8 \mathrm{~Gy}$, dose rate I Gy/min) or left untreated $(0 \mathrm{~Gy})$ and $8 \mathrm{hr}$ later cells were subjected to real-time PCR assay for detection of miR-34a level. (D). HCTII 6 p53 $3^{+/+}$cells and HCTII 6 p53\%- cells were irradiated with 4 Gy (dose rate I Gy/min) irradiation and $0,4,8,24,48 \mathrm{hr}$ later cells were subjected to real-time PCR assay for detection of miR-34a level. *:P < 0.05 . 
This result was consistent with the previous concept that p53 enhanced radio-sensitivity. Furthermore, it is consistent with the radio-sensitivity difference between these two cell lines. The relative level of miR-34a was also higher in HCT116 p53+/+ cells than in HCT116 p53\% cells (Figure 3C, 0Gy). Also, miR-34a was induced by IR in a dose and time

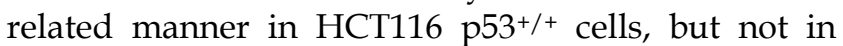
HCT116 p53\%- cells (Figure 3C and 3D). These data indicate that miR-34a is positively correlated to cellular radio-sensitivity and the induction of miR-34a was in a p53 dependent manner.

\section{MiR-34a maybe a critical mediator of radiation injury and may also be a critical target of radio- protection}

Since miR-34a is closely related to age and tissue related radio-sensitivity, studies have shown that in-
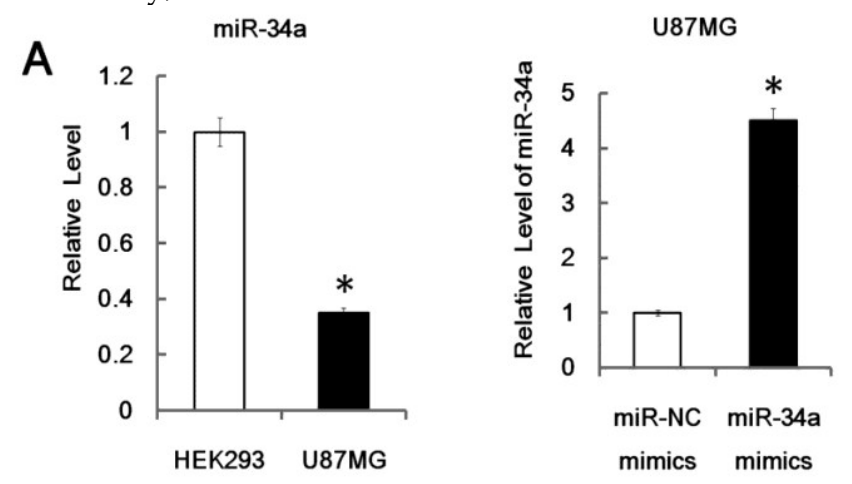

activation of miR-34a strongly attenuates apoptosis in cells, whereas over-expression of miR-34a increases apoptosis [11]. Next we posited that miR-34a may have significant function on radiation-induced cell death. To prove our theory, we used in vitro transfection assays to explore the biological effects of miR-34a in radiation biology. Human glioblastoma cell U87MG cells and human embryonic kidney HEK293 cells were used for transfection. We found that U87MG cells have a relatively lower miR-34a expression level than HEK293 cells and transient transfection of miR-34a mimics could lead to substantial increased production of mature miR-34a in U87MG cells, while, transient transfection of miR-34a specific inhibitors (anti-34a) could partly knockdown the production of miR-34a in 293 cells, as can be detected by qRT-PCR (Figure 4 A).

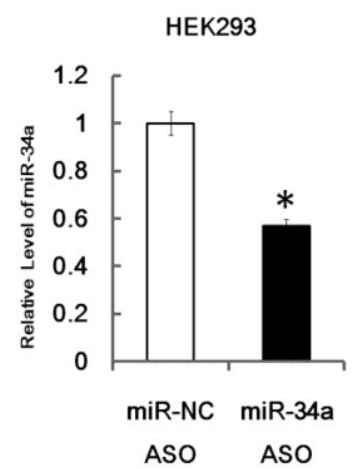

B

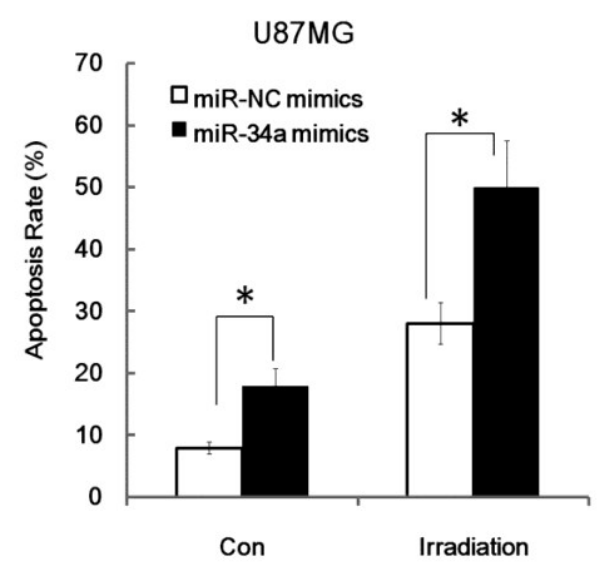

C

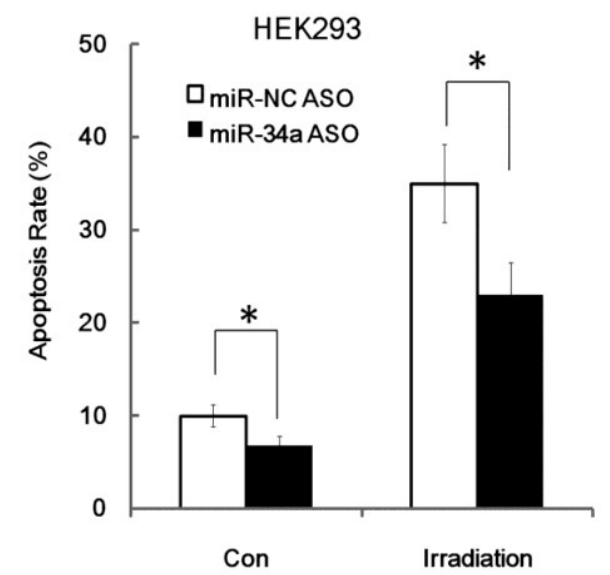

Figure 4. MiR-34a enhanced radiation induced apoptosis. (A) Relative miR-34a expression level in HEK293cells and U87MG cells and validation of miR-34a mimics (miR-34a) and miR-34a specific inhibitors (miR-34a ASO, antisense oligo). MiR-34a mimics and miR-34a ASO were transfected with nonspecific control miRNA mimics (NC) and NC ASO in to U87MG cells and HEK293cells as indicated for 24 hours, the relative levels of miR-34a were analyzed by qRT-PCR. (B) U87MG cells were transfected with nonspecific control NC or miR-34a for 48 hours and then subjected to radiation (+IR; 7Gy, dose rate I $\mathrm{Gy} / \mathrm{min}$ ) or left untreated. After an additional 24 hours cells were stained with annexin V-FITC and PI and subjected to FACS assay of cellular apoptosis. (C) HEK 293 cells were transfected with miR-34a specific inhibitors (miR-34a ASO) or scrambled 
miRNA control (NC ASO) for $48 \mathrm{hr}$ later. Then cells were exposed to irradiation (+IR; 7Gy, dose rate I Gy/min) or left untreated (con). After an additional 24hours cells were stained with annexin $\mathrm{V}$ and $\mathrm{Pl}$ and subjected to FACS assay of cellular apoptosis. Data are representative of at least three wells. *:P $<0.05$.

More importantly, we found that over-expression of miR-34a mimics markedly suppressed cell proliferation and enhanced apoptosis in transfected U87MG cells both before and after IR (Figure 4B, apoptosis; proliferation data not shown). Furthermore, we found that inhibition of miR-34a expression could markedly decrease the apoptosis rate of HEK293 cells after 7Gy irradiation (Figure 4C).

Similarly, over-expression of miR-34a mimics markedly enhanced the radio-sensitivity of HCT116 p53/cells while inhibition of miR-34a expression could markedly decrease the radio-sensitivity of HCT116

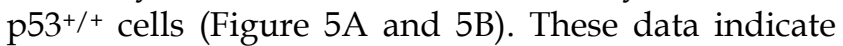
that miR-34a maybe a critical mediator of radiation injury and may also be a critical target of radioprotection.

A

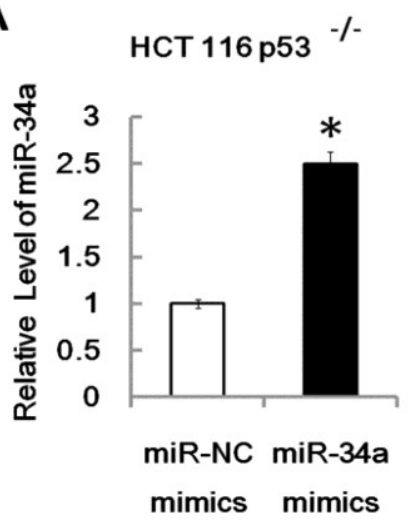

B

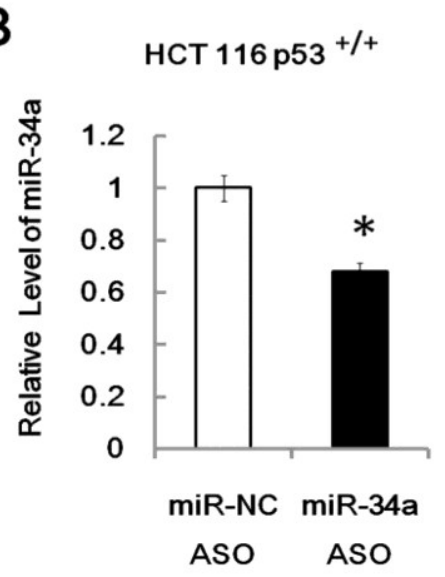

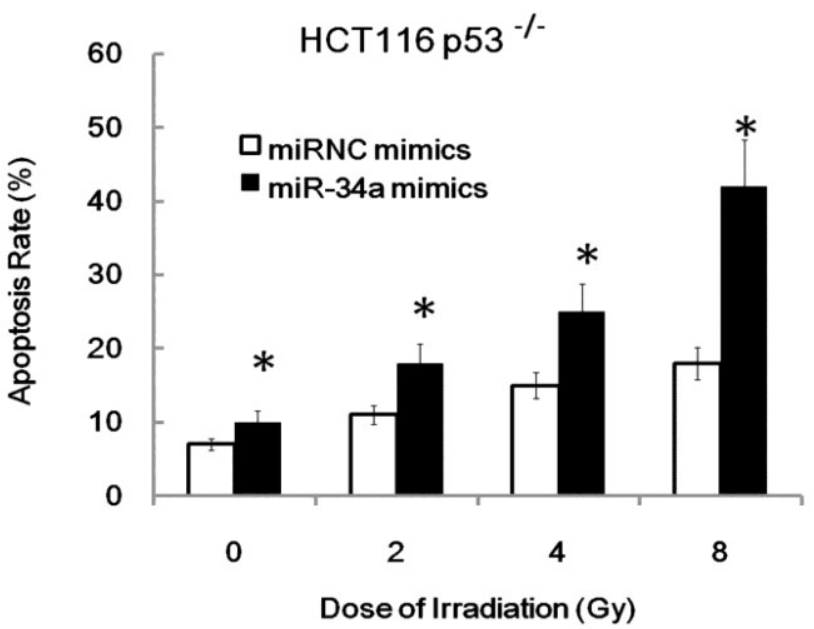

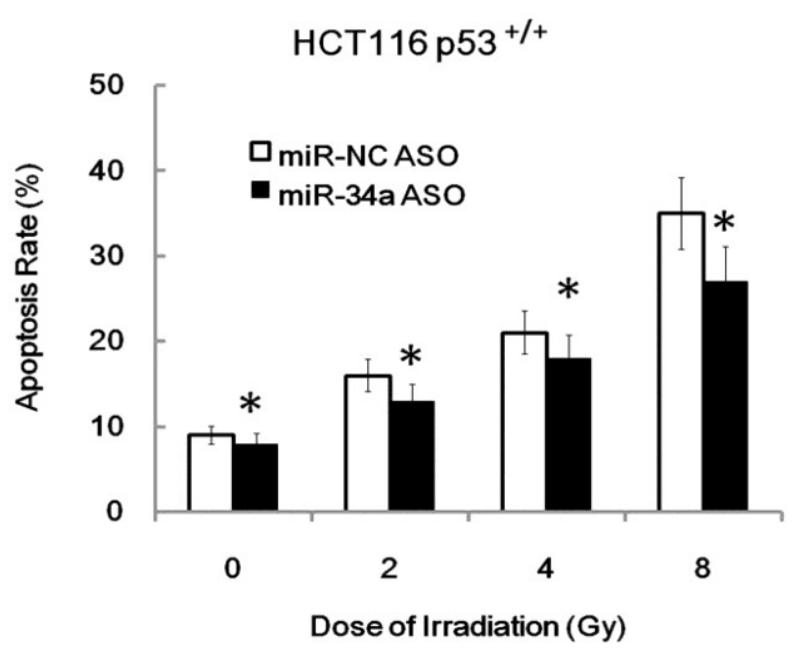

Figure 5.Over-expression of miR-34a mimics markedly enhanced the radio-sensitivity of HCTII $6 \mathrm{p}^{-/}$cells while inhibition of miR-34a expression could markedly decrease the radio-sensitivity of HCTII6 p5 $3^{+/+}$cells. *: P < 0.05 ; NS: No Significant difference detected. 


\section{One possible downstream target of miR-34 that leads to different radio-sensitivity was $\mathrm{Bcl}-2$}

MiRNAs function through binding and inhibition of the 3'UTR of their target genes [4, 5]. A couple of published targets (e.g. cyclin-dependent kinase (cdk) 4, cyclin E2, cMet, cdk6, and bcl-2) of miR-34a were identified by many groups $[13,14]$. These molecules have been proven to play a critical role in apoptosis, cell survival and cell cycle. We are particularly interested in Bcl-2 because many studies have shown that the Bcl-2 family proteins are key molecules that modulate radio-sensitivity in human cancer cells [33], specific, over-expression of bcl-2 has been proven to reduce the radio-sensitivity of many cancer cell lines [34] and Bcl-2 has been proven to be a direct target of miR-34a. To explore the relevance of Bcl-2 in miR-34a related radio-sensitivity, we performed Bcl-2 KnockDown (KD) study. Three anti-Bcl-2 siRNA (siRNA 1, siRNA 2 and siRNA 3) and a control non-targeting

\section{A}

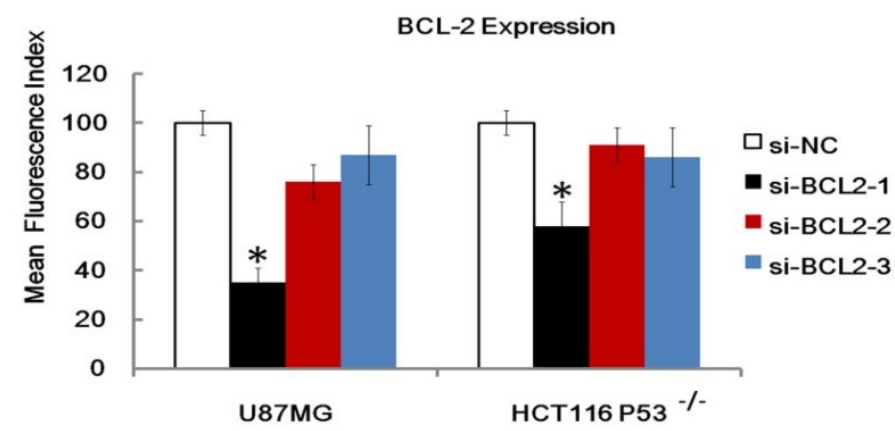

Figure 6. One possible downstream target of miR-34a that leads to different radio-sensitivity was Bcl-2. (A). Validation of three anti-Bcl-2 siRNA (siRNA 1, siRNA 2 and siRNA 3) and a control non-targeting siRNA (siRNA NC) in transfected into U87MG cells and HCTII 6 p53 cells 48 hours after transfection. (B). Knockdown of $\mathrm{Bcl}-2$ (si-Bcl-2-1), but not KD of nonspecific control (si-NC), enhanced the apoptosis rate of the HCTII 6 p53 $3^{-1-}$ cells and U87MG cells after IR, which mimics the effects of miR34a over-expression. (C). Knockdown of $\mathrm{Bcl}-2$ (si-Bcl-2) attenuated, but not fully wiped off, the radio-protective role of miR34a inhibitor in HEK293 cells and HCTII 6 p53 $3^{+/+}$cells. *: $\mathrm{P}<0.05$.
siRNA (siRNA NC) were transfected into U87MG cells and HCT116 p53-/- cells and we found that antiBcl-2 siRNA 1 showed the highest Bcl-2 knockdown capacity as detected by FACS assay (Figure 6A). We also found that KD of Bcl-2 (Bcl-2-KD), but not KD of nonspecific control (NC-KD), enhanced the apoptosis rate of the HCT116 p53\%- cells and U87MG cells after IR, which mimics the effects of miR-34a over-expression (Figure 6B). Furthermore, KD of Bcl-2 also attenuated, but not fully wiped off the radio-protective role of miR-34a inhibitor in HEK293 cells and HCT116 p53+/+ cells (Figure 6C). These data, taken together, indicate that although other possible downstream targets existed, one possible downstream target of miR-34a that leads to different radio-sensitivity was Bcl-2.

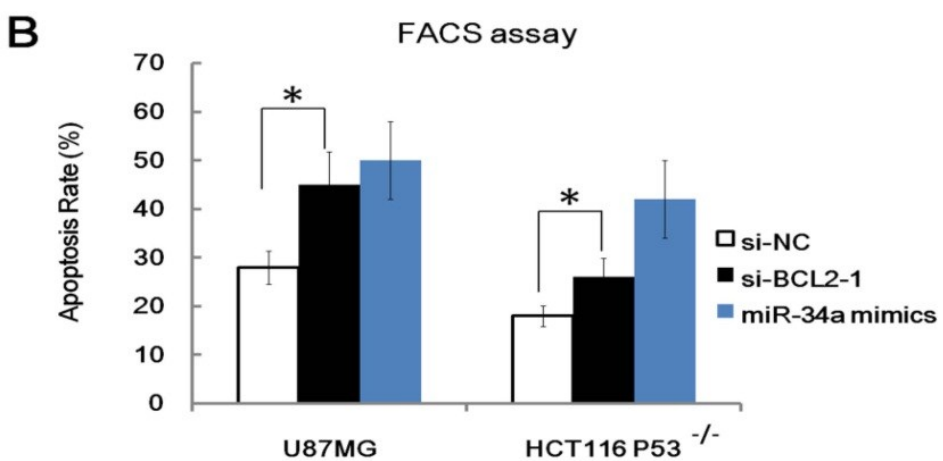

C

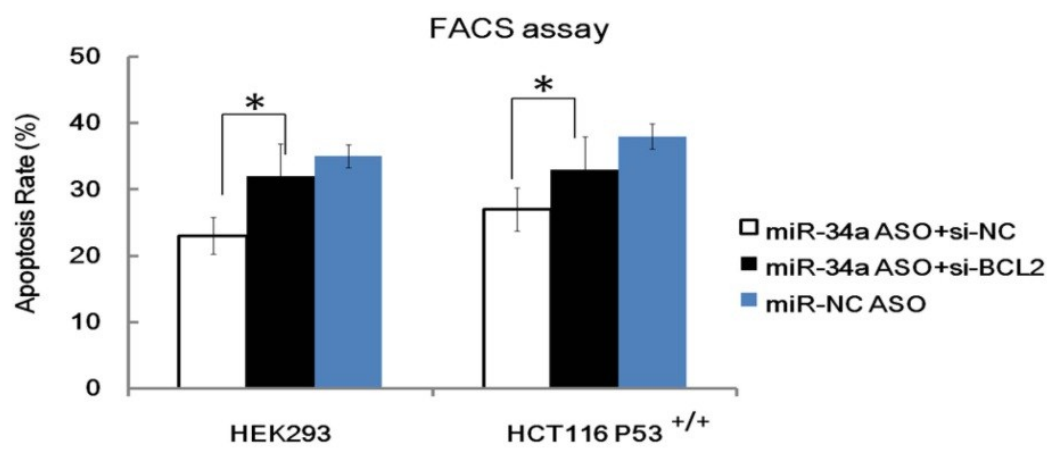




\section{Serum miR-34a as a Novel Indicator of Radiation injury}

It is of critical importance to develop novel biomarkers or indicators for individual radiation biodosimetry of exposure to ionizing radiation [35, 36]. However, the potential miRNA indicators in radiation biology are largely ignored. Recent studies have shown that miRNAs are novel myocardial biomarker, and potential biomarkers for colorectal cancer and many other types of human cancers [37-39]. More importantly, some recent studies demonstrated that plasma or serum miRNAs are potential biomarkers for some diseases [37-39]. We asked whether plasma or serum miR-34a can serve as a biomarker or indicator of radiation injury. To explore this issue, we conducted a q-PCR assay to detect the relative miR-34a level in different mice before and after 7Gy of TBI. Consistently, we used exogenous U6 RNA as loading control and found that serum miR-34a, but not serum miR-16, was enhanced after irradiation (Figure 7A).

We also found that miR-34a can be rapidly induced in serum from irradiated mice but not non-irradiated control mice, and the

Figure 7. Serum miR-34a is enhanced after Radiation injury. (A). Adult BALB/c mice were either irradiated with different doses (+IR; 2 Gy, 7 Gy or $12 \mathrm{~Gy}$, dose rate I Gy/min) or left untreated (con) and sacrificed $8 \mathrm{hr}$ later, Serum RNA was subjected to qRT-PCR analysis. Levels of miR-34a and miR-16 were normalized to exogenous U6 RNA. Data show the relative miR-34a and miR-16 expression level. $N=6$. (B) Yong or adult $B A L B / c$ mice were either irradiated (+IR; 7Gy, dose rate I Gy/min) or left untreated (con) and sacrificed $24 \mathrm{hr}$ later. Serum total RNA was subjected to qRT-PCR analysis. Levels of miR-34a were normalized to miR-16 in the same sample. Data show the relative miR-34a expression level. $N=6$. (C) Yong or adult $B A L B / c$ mice were either irradiated (+IR; 7Gy, dose rate I Gy/min) or left untreated (con) and sacrificed 4,8,24 and $48 \mathrm{hr}$ later, Serum RNA was subjected to qRT-PCR analysis for detection of miR-34a. $\mathrm{N}=6$. (D) Yong or adult BALB/c mice were either irradiated with different doses (+IR; $2 \mathrm{~Gy}, 7$ Gy or $12 \mathrm{~Gy}$, dose rate I Gy/min) or left untreated (con) and sacrificed $8 \mathrm{hr}$ later, Serum RNA was subjected to QRT-PCR analysis for detection of miR-34a.Levels of miR-34a were normalized in the same sample and the level of miR-34a in adult un-irradiated mice was regarded as $100 \%$. Data show the relative miR-34a expression level. $\mathrm{N}=6$. $*: P<0.05 ;$ NS: No Significant difference detected.
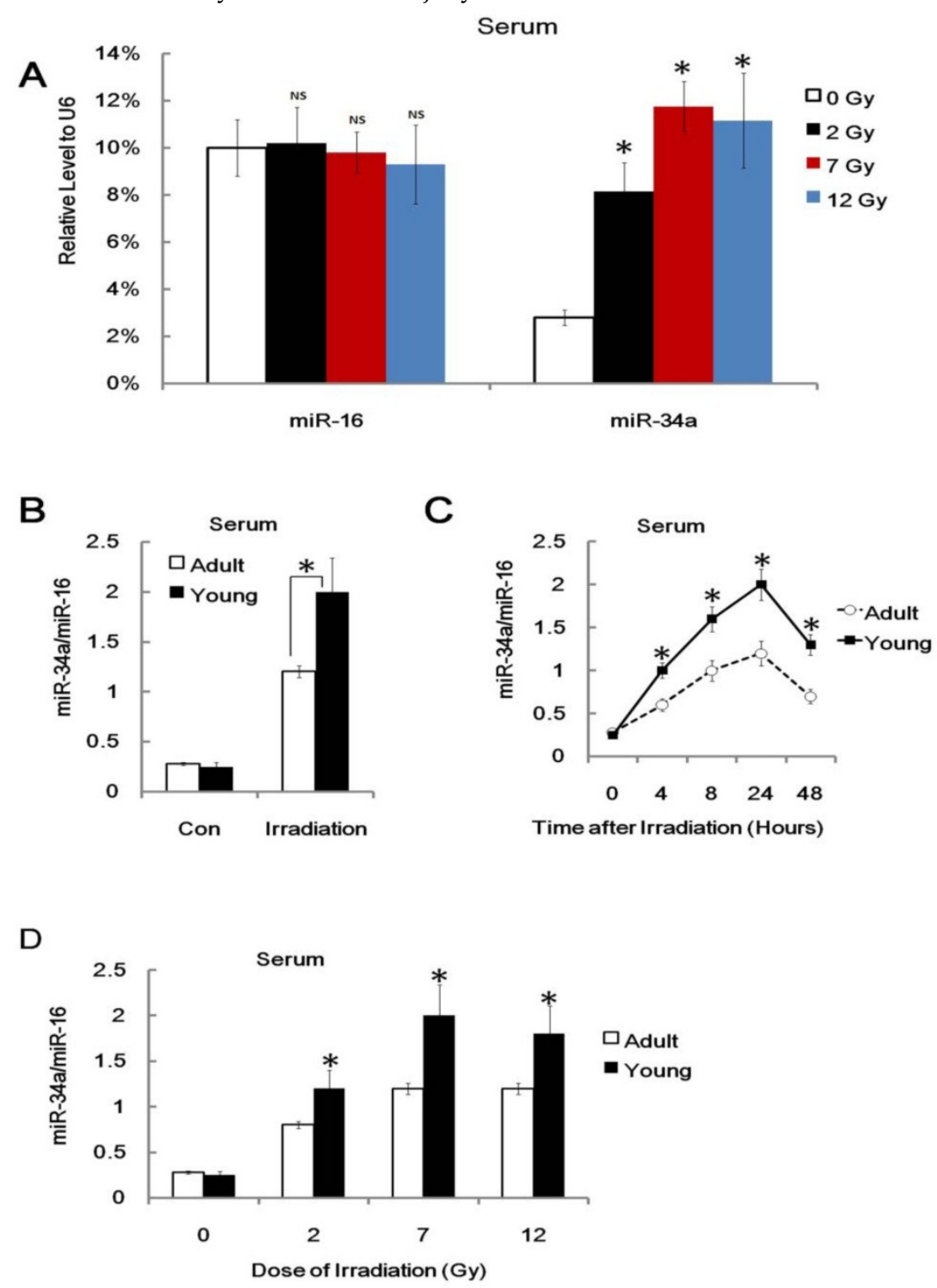


\section{Discussion}

Over 20 percent of cancer patients will require radiation therapy during the treatment of their disease. Radiation can induce significant damage and the development of radioprotectants for medical and bio-defense applications are very important [22, 23, $40,41]$. Studies also show that miRNAs play pivotal roles in diverse processes such as development, differentiation, cell proliferation, stress response and cancer $[8,9,42,43]$. However, there has been very limited study demonstrating the role of miRNA in radio-sensitivity in vivo and in vitro. Additionally, radiation damage biomarkers or indicators in miRNA levels are not well understood.

In this study, we first found that miR-34a could be induced in many organs by radiation of both young and adult mice. However, the level of miR-34a induced by young mice was much higher compared to adult mice, which was closely correlated with the fact that young mice had higher radio-sensitivity when compared to adult mice. Next, we found that miR-34a correlated closely with radio-sensitivity variations of different tissues in genetic normal mice. However, the level of miR-34a induced by IR seems to be in a p53 dependent manner since the level of miR-34a induced by IR in HCT116 p53\% cells was decreased significantly when compare to HCT116 p53 $3^{+/+}$cells. Then, we used transfection assay to find that over-expression of miR-34a markedly suppressed cell proliferation and enhanced apoptosis in transfected U87MG and HCT116 p53\%- cells both before and after radiation. Furthermore, We also found that the possible downstream target of miR-34 that lead to different radio-sensitivity was $\mathrm{Bcl}-2$ since over-expression of miR-34 and knockdown of Bcl-2 could significantly enhance the radio-sensitivity of different cells. Finally, we found that miR-34a could be detected not only in tissues, but also in mice serum, while the p53 mRNA could not be detected in mice serum after IR, which indicated miR-34a as a novel biomarker of radiation injury. Taken together, our data demonstrated that miR-34a may be a novel indicator, mediator and target of radiation injury, radio-sensitivity and radioprotection.

In summary, this study indicates that miR-34a has the potential to be a novel mediator and target of radiation injury, radio-sensitivity and radioprotection.

\section{Acknowledgment}

We thank Dr. Cindy Savage and Trip Barthel, M.A. for their assistance in editing the manuscript.
Special thanks to Dr. Xuetao Cao, Dr. Yushi Yao \& Dr. Sheng $\mathrm{Xu}$ from National Key Laboratory of Medical Immunology and Dr. Pu You from department of cell biology of our university for providing additional helps. We wish to thank Dr. Min Li from the department of hygienics of our university for doing the q-RT-PCR assays for us.

This work was supported in part by the grants from National Natural Science Foundation of China (No. 31070762 \& No. 31070761). The funders had no role in study design, data collection and analysis, decision to publish, or preparation of the manuscript.

\section{Conflict of Interests}

The authors have declared that no conflict of interest exists.

\section{References}

1. Bartel D.P. MicroRNAs: genomics, biogenesis, mechanism, and function. Cell, 2004. 116(2): 281-97.

2. Lewis B.P, et al. Prediction of mammalian microRNA targets. Cell, 2003. 115(7): 787-98.

3. Ruby J.G, Jan C.H., and Bartel D.P. Intronic microRNA precursors that bypass Drosha processing. Nature, 2007. 448(7149): 83-6.

4. Lim L.P, et al. Microarray analysis shows that some microRNAs downregulate large numbers of target mRNAs. Nature, 2005. 433(7027): 769-73.

5. Hornstein E, et al. The microRNA miR-196 acts upstream of Hoxb8 and Shh in limb development. Nature, 2005. 438(7068): 671-4.

6. Deng Y, et al. MicroRNA-137 Targets Carboxyl-terminal Binding Protein 1 in Melanoma Cell Lines. Int J Biol Sci, 2011. 7(1): 133-7.

7. Guo $\mathrm{H}$, et al. Mammalian microRNAs predominantly act to decrease target mRNA levels. Nature. 2010;466(7308): 835-40.

8. Chen C.Z, et al. MicroRNAs modulate hematopoietic lineage differentiation. Science, 2004. 303(5654): 83-6.

9. Giraldez A.J, et al. MicroRNAs regulate brain morphogenesis in zebrafish. Science, 2005. 308(5723): 833-8.

10. Lim L.P, et al. Vertebrate microRNA genes. Science, 2003. 299(5612): 1540.

11. He L, et al. A microRNA component of the p53 tumour suppressor network. Nature, 2007. 447(7148): 1130-4.

12. Jaklevic B, et al. Modulation of ionizing radiation-induced apoptosis by bantam microRNA in Drosophila. Dev Biol, 2008. 320(1): 122-30.

13. Ishii $\mathrm{H}$ and Saito T. Radiation-induced response of micro RNA expression in murine embryonic stem cells. Med Chem, 2006. 2(6): 555-63.

14. Ilnytskyy $\mathrm{Y}$, et al. Altered microRNA expression patterns in irradiated hematopoietic tissues suggest a sex-specific protective mechanism. Biochem Biophys Res Commun, 2008. 377(1): 41-5.

15. El-Deiry W.S. The role of p53 in chemosensitivity and radiosensitivity. Oncogene, 2003. 22(47): 7486-95.

16. Abdulkarim B, et al. Antiviral agent Cidofovir restores p53 function and enhances the radiosensitivity in HPV-associated cancers. Oncogene, 2002. 21(15): 2334-46.

17. Siles E, et al. Relationship between p53 status and radiosensitivity in human tumour cell lines. Br Cancer J, 1996. 73(5): 581-8. 
18. Shiomitsu K, et al. Radiosensitivity of canine osteosarcoma cells transfected with wild-type p53 in vitro. Vet Comp Oncol, 2008. 6(3): 193-200.

19. Maebayashi K, et al. p53 mutation decreased radiosensitivity in rat yolk sac tumor cell lines. Int J Radiat Oncol Biol Phys, 1999. 44(3): 677-82.

20. Shin H.J, et al. Human papillomavirus 16 E6 increases the radiosensitivity of p53-mutated cervical cancer cells, associated with up-regulation of aurora A. Int J Radiat Biol, 2010. 86(9): 769-79.

21. Abrams H.L. Influence of age, body weight, and sex on susceptibility of mice to the lethal effects of X-radiation. Proc Soc Exp Biol Med, 1951. 76(4): 729-32.

22. Sacher G.A. Dependence of acute radiosensitivity on age in adult female mouse. Science, 1957. 125(3256): 1039-40.

23. Crosfill M.L, Lindop P.J., and Rotblat J. Variation of sensitivity to ionizing radiation with age. Nature, 1959. 183(4677): 1729-30.

24. Lindop P.J and Rotblat J. The age factor in the susceptibility of man and animals to radiation. I. The age factor in radiation sensitivity in mice. Br Radiol J, 1962. 35: 23-31.

25. Doll R. The age factor in the susceptibility of man and animals to radiation. II. Age differences in susceptibility to carcinogenesis in man. Br Radiol J, 1962. 35: 31-6.

26. Chaffey J.T and Hellman S. Differing responses to radiation of murine bone marrow stem cells in relation to the cell cycle. Cancer Res, 1971. 31(11): 1613-5.

27. Qian L, et al. Radioprotective effect of hydrogen in cultured cells and mice. Free Radic Res, 2010. 44(3): 275-82.

28. Wang G.K, et al. Circulating microRNA: a novel potential biomarker for early diagnosis of acute myocardial infarction in humans. Eur Heart J, 2010. 31(6): 659-66.

29. Li L.M, et al. Serum microRNA profiles serve as novel biomarkers for infection HBV and diagnosis of HBV-positive hepatocarcinoma. Cancer Res, 2010. 70(23): 9798-807.

30. Chen X, et al. Characterization of microRNAs in serum: a novel class of biomarkers for diagnosis of cancer and other diseases. Cell Res, 2008. 18(10): 997-1006.

31. Hu Z, et al. Serum microRNA signatures identified in a genome-wide serum microRNA expression profiling predict survival of non-small-cell lung cancer. J Clin Oncol, 2010. 28(10): 1721-6.

32. Hao J, et al. MicroRNA 483-3p suppresses the expression of DPC4/Smad4 in pancreatic cancer. Lett FEBS, 2011. 585(1): 207-13.

33. Streffer J.R, et al. BCL-2 family proteins modulate radiosensitivity in human malignant glioma cells. Neurooncol J, 2002. 56(1): 43-9.

34. Ahmed M.M, et al. Restoration of transforming growth factor-beta signaling enhances radiosensitivity by altering the Bcl-2/Bax ratio in the p53 mutant pancreatic cancer cell line MIA PaCa-2. J Biol Chem, 2002. 277(3): 2234-46.

35. Marchetti F, et al. Candidate protein biodosimeters of human exposure to ionizing radiation. Int J Radiat Biol, 2006. 82(9): 605-39.

36. Susskind $\mathrm{H}$, et al. Increased plasma levels of matrix metalloproteinase- 9 and tissue inhibitor of metalloproteinase- 1 in lung and breast cancer are altered during chest radiotherapy. Int J Radiat Oncol Biol Phys, 2003. 56(4): 1161-9.

37. $\mathrm{Ng}$ E.K, et al. Differential expression of microRNAs in plasma of patients with colorectal cancer: a potential marker for colorectal cancer screening. Gut, 2009. 58(10): 1375-81.

38. Schetter A.J and Harris C.C. Plasma microRNAs: a potential biomarker for colorectal cancer? Gut, 2009. 58(10): 1318-9.

39. Chim S.S, et al. Detection and characterization of placental microRNAs in maternal plasma. Clin Chem, 2008. 54(3): 482-90.
40. ICRP. The 2007 Recommendations of the International Commission on Radiological Protection. ICRP publication 103. Ann ICRP, 2007. 37(2-4): 1-332.

41. Burdelya L.G, et al. An agonist of toll-like receptor 5 has radioprotective activity in mouse and primate models. Science, 2008. 320(5873): 226-30.

42. Mayr C, Hemann M.T., and Bartel D.P. Disrupting the pairing between let-7 and Hmga2 enhances oncogenic transformation. Science, 2007. 315(5818): 1576-9.

43. Bartel D.P and Chen C.Z. Micromanagers of gene expression: the potentially widespread influence of metazoan microRNAs. Nat Rev Genet, 2004. 5(5): 396-400. 